\title{
Response of Ictal Asystole to Pacemaker Implantation Documented by Video-EEG/ECG
}

\author{
Mona AlKhawajah, David B. MacDonald, Abdulaziz AlSemari, Ibrahim AlThubaiti, \\ Donald McLean
}

Can J Neurol Sci. 2013; 40: 606-608

Asystole is a rare and potentially fatal manifestation of partial seizures. We present the electroclinical features of a patient with epileptic ictal asystole documented by video electroencephalogram/electrocardiogram (EEG/ECG) monitoring before and after pacemaker implantation.

\section{CASE}

A 30-year-old left-handed male computer operator suffering from clinically diagnosed dyscognitive (complex partial) seizures with secondary generalization since age 24 was admitted for epilepsy monitoring. His attacks consisted of an aura of "flashes from past memory" followed by loss of awareness with amnesia and often progressed to generalized tonic-clonic motor activity. They continued in clusters of four to five attacks about every two weeks despite phenytoin and carbamazepine therapy as well as trials of topiramate and levetiracetam.

He reported a closed head injury with loss of consciousness while playing soccer at age ten. There was no history of other risk factors or febrile convulsions. Past health, functional enquiry and family history were unremarkable. In particular, there were no other neurologic or cardiac symptoms and there was no family history of epilepsy or heart disease.

Neurological examination was normal. A routine outpatient EEG had shown bilateral intermittent slow activity maximal in the left temporal region. Magnetic resonance imaging (MRI) disclosed right inferior frontal lobe atrophy and gliosis suspected to be an old traumatic lesion; no temporal lobe abnormalities were evident. Positron emission tomography suggested right inferior frontal and bilateral mesial temporal hypometabolism. Neuropsychologic testing suggested mild non-dominant frontal and bitemporal deficits.

Video-EEG/ECG monitoring disclosed occasional left temporal spikes or sharp waves and rare independent right temporal sharp waves. Nine seizures were captured and all began with a left temporal delta frequency seizure pattern. Two produced aura followed by motionlessness and amnesia with bradycardia but no asystole. Seven showed bradycardia rapidly progressing to asystole several seconds after EEG onset. Two of these occurred during sleep and produced less than eight second asystole with no clinical signs. The other five occurred during wakefulness and produced symptomatic asystole of 8-21 seconds duration. Each began with a left temporal seizure pattern followed by aura, motionlessness and then asystole. After a few seconds of asystole the patient collapsed unconscious with brief clonic jerking and then tonic neck and limb extension (Figure 1).

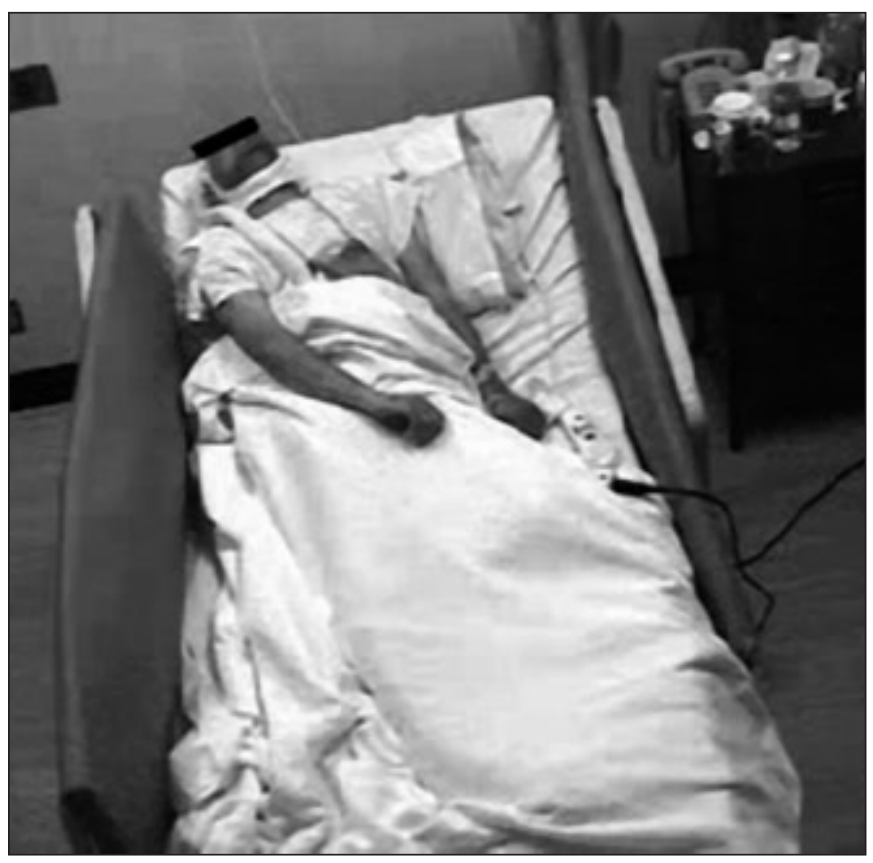

Figure 1: Tonic neck and limb extension with arm pronation during epileptic ictal asystole (corresponds to Figure 2E).

The corresponding EEG showed transient diffuse slow activity followed by attenuation and superimposed EMG artifact; a predominantly left temporal seizure pattern persisted and appeared to slow down (Figure 2). A few seconds after the reappearance of sinus rhythm the patient began to regain consciousness after brief post-ictal confusion. The corresponding EEG showed transient diffuse slow activity accompanied by seizure pattern termination and then resolution to background rhythms after brief post-ictal left temporal polymorphic delta activity (Figure 2).

From the Section of Neurology (MA, AA, DM), Section of Clinical Neurophysiology (DBM), Section of Neurosurgery (IA), Department of Neurosciences, King Faisal Specialist Hospital \& Research Center, Riyadh, Saudi Arabia.

Received November 27, 2012. Final Revisions Submitted January 15, 2013 Correspondence to: David B. MacDonald, Section of Clinical Neurophysiology, Department of Neurosciences, King Faisal Specialist Hospital \& Research Center, MBC 76, PO Box 3354, Riyadh, 11211, Saudi Arabia. Email: dbmacdon@yahoo.com. 


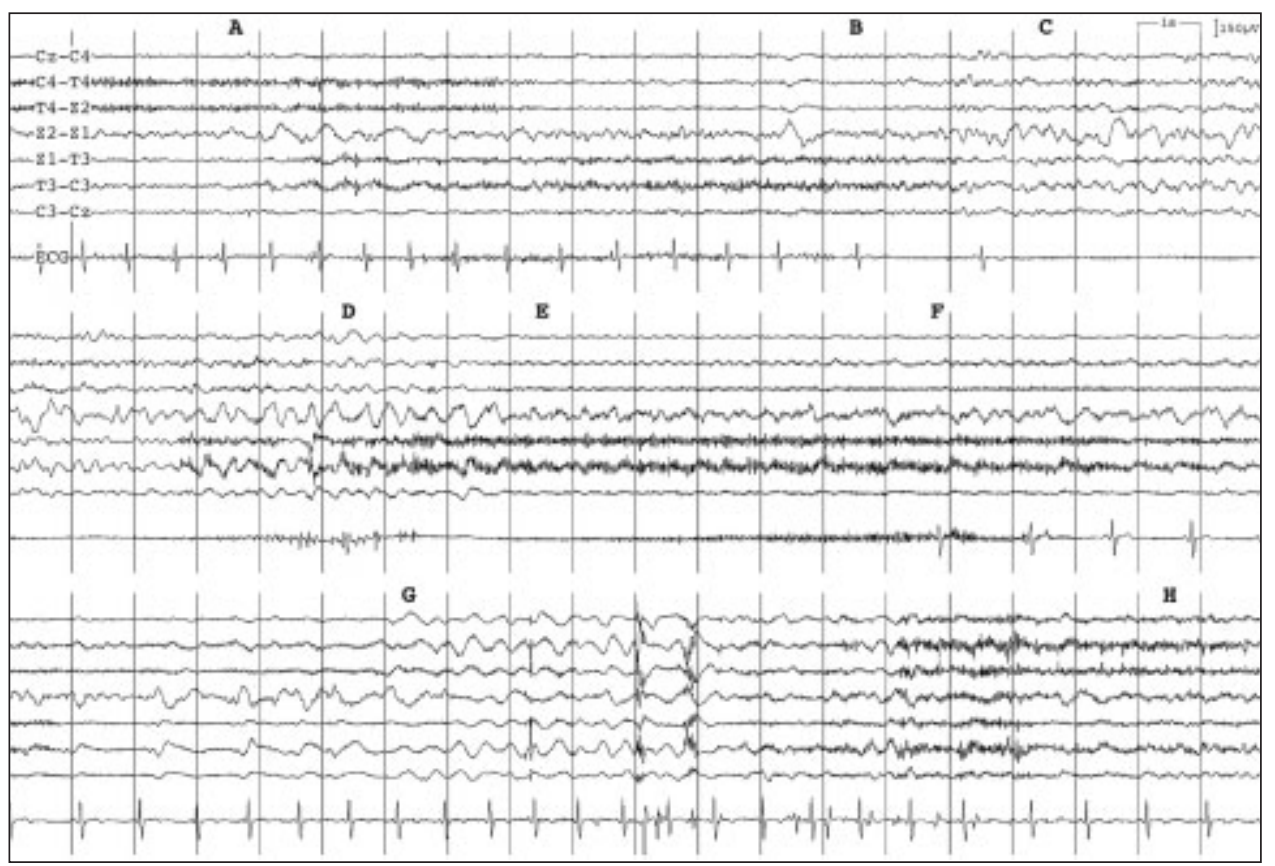

Figure 2: Epileptic ictal asystole. Z1 and Z2: left and right zygomatic electrodes. An abbreviated 7-channel coronal bitemporal EEG montage is displayed for clarity; full montages showed the same result. A: Left temporal seizure onset followed by aura, button-press and then motionlessness. B: Bradycardia. C: Asystole. D: Transient diffuse slow activity with collapse and clonic jerking. E: Generalized attenuation accompanied by tonic motor activity (see Figure 1). There is corresponding EMG artifact in $T 3$ and ECG channels. A predominantly left temporal seizure pattern continues, but appears to slow down. F: Sinus rhythm recovery followed by body relaxation. G: Transient diffuse rhythmic delta activity and seizure pattern termination. H: Recovery of EEG background and consciousness after brief post- left temporal polymorphic delta and confusion.
Cardiologic consultation and investigation found no primary heart disease, dysrhythmia or conduction disturbance. The diagnosis was changed to dyscognitive seizures with ictal asystole-convulsive syncope. A demand cardiac pacemaker was implanted. At follow-up he continued to have seizures consisting of aura followed by loss of awareness and amnesia, but the attacks no longer progressed to collapse or convulsive activity.

Repeat video-EEG/ECG monitoring captured twelve seizures consisting of aura followed by motionlessness and amnesia without collapse or convulsive activity. The EEG again showed a predominantly left temporal seizure pattern, but no transient diffuse slow activity or attenuation and the ECG showed paced rhythm shortly after seizure onset (Figure 3).

Intracarotid amytal testing revealed left hemisphere language dominance and intact memory scores on each side. Intracranial EEG monitoring with right frontal and bitemporal subdural strips and bilateral hippocampal depth electrodes disclosed independent right orbitofrontal and bilateral hippocampal interictal spikes. Electrocardiogram was not included. Each of eight ictal recordings suggested right orbitofrontal seizure onset with rapid initial spread to either the left (four) or right (four) hippocampus. Unfortunately, resection of the right inferior frontal lesion and orbitofrontal cortex had no beneficial effect on the dyscognitive seizures. The pacemaker remains in place and the attacks still do not progress to collapse or convulsive activity.

\section{DISCUSSION}

Cardiac manifestations of partial seizures commonly include tachycardia, but bradycardia is infrequent and asystole is rare. A few previous case reports and short series describe the epileptic ictal bradycardia or asystole syndrome that occurs more often in men than women and mainly with temporal, less often with frontal and very rarely with other focal seizures. ${ }^{1,2}$ Although a preponderance of left hemispheric epileptic foci has been suggested, there is no consistent lateralization. ${ }^{1}$ We found only one previous case report illustrating video-EEG/ECG recordings before and after pacemaker implantation. ${ }^{2}$ Our report may therefore add to the understanding of this disorder and its management.

The recordings indicated that our patient's original attacks were a combination of dyscognitive seizures with ictal asystole producing ischemic EEG changes and convulsive syncope. This formulation matches the known manifestations of syncope that uniformly consist of diffuse EEG slowing followed by attenuation accompanied by loss of consciousness and sometimes convulsive movements due to acute brain ischemia and then diffuse slow activity resolving to normal background with recovery of consciousness as cerebral perfusion resumes. ${ }^{3}$ As in our patient, the convulsive activity typically involves clonic jerking and/or tonic extension resembling decerebrate posturing and is easily mistaken for epileptic convulsions. ${ }^{3}$ The corresponding EEG often contains electromyelogram (EMG) artifact that has sometimes been misinterpreted as a highfrequency seizure pattern. ${ }^{3}$ With syncope due to epileptic ictal asystole, a slowing focal seizure pattern may persist during the diffuse slow-attenuation-slow EEG sequence of syncope. ${ }^{3}$ That our patient's seizure pattern ended with the final burst of slow activity corresponds to a previous report suggesting that cerebral ischemia during asystole might actually terminate the inciting seizure. $^{4}$

Because ictal asystole is a suspected mechanism of sudden unexpected death in epilepsy and can cause traumatic falls or possibly ischemic brain damage, pacemaker implantation has been recommended for refractory patients. ${ }^{5}$ Our report graphically illustrates the protective response to cardiac pacing, which modified our patient's attacks by preventing the asystole- 


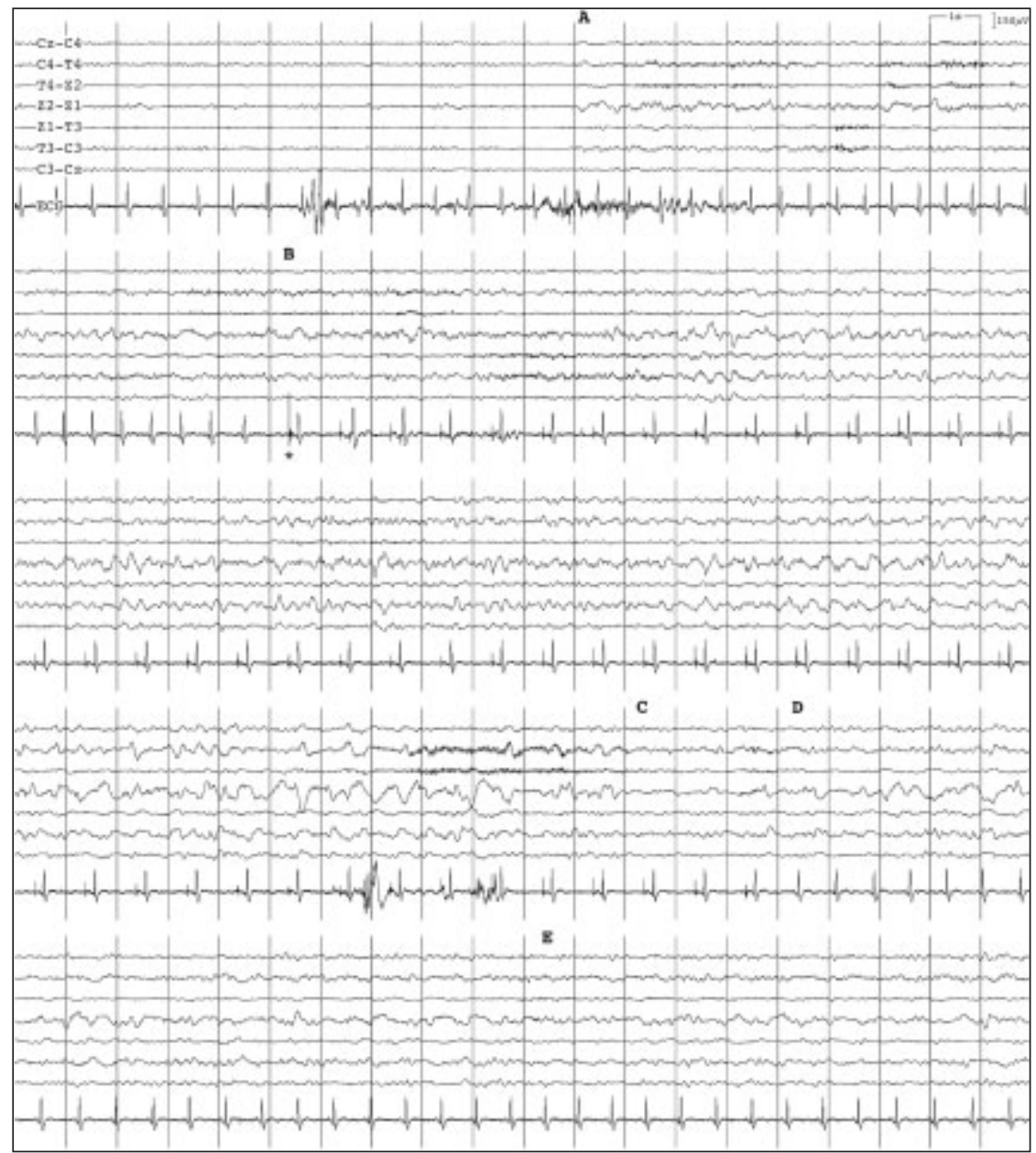

Figure 3: Ictal pacemaker activation. Z1 and Z2: left and right zygomatic electrodes. A: Left temporal seizure onset followed by aura, button-press and then motionlessness. B: Paced cardiac rhythm with pacemaker artifact (*) preceding each QRS complex. C: Seizure termination. D: Sinus rhythm recovery. E: Post-ictal left temporal polymorphic delta activity and confusion.

convulsive syncope component, but of course, did not stop his dyscognitive seizures.

Had the subsequent epilepsy surgery eliminated his seizures, we could have eventually considered pacemaker removal. Since it did not, the pacemaker remains in place and continues to provide asystole protection. This is relevant because the alternative strategy of proceeding directly to epilepsy surgery to eliminate the seizures can avoid a pacemaker when successful. ${ }^{5}$ However, postoperative seizure freedom is never certain and those patients who do experience recurrence would be left without protection against asystole. Consequently, pacemaker implantation as soon as the diagnosis is made may be the safer approach.

Finally, the recordings illustrate the value of video-EEG/ECG monitoring for the otherwise difficult differentiation between partial seizures with secondary generalization and partial seizures with ictal asystole-convulsive syncope. ${ }^{3}$

\section{REFERENCES}

1. Britton JW, Ghearing GR, Benarroch EE, Cascino GD. The ictal bradycardia syndrome: localization and lateralization. Epilepsia. 2006;47(4):737-44.

2. Strzelczyk A, Bauer S, Knake S, Oertel WH, Hamer HM, Rosenow F. Ictal asystole in temporal lobe epilepsy before and after pacemaker implantation. Epileptic Disord. 2008;10(1):39-44.

3. Brenner RP. Electroencephalography in syncope. J Clin Neurophysiol. 1997;14(3):197-209.

4. Schuele SU, Bermeo AC, Alexopoulos AV, Burgess RC. Anoxiaischemia: a mechanism of seizure termination in ictal asystole. Epilepsia. 2010;51(1):170-3.

5. Strzelczyk A, Cenusa M, Bauer S, et al. Management and long-term outcome in patients presenting with ictal asystole or bradycardia. Epilepsia. 2011;52(6):1160-7. 